\title{
Interactions between AdS black hole molecules
}

\author{
Suvankar Dutta $\odot^{*}$ and Gurmeet Singh Punia $\odot^{\dagger}$ \\ Indian Institute of Science Education \& Research Bhopal, Bhopal Bypass, Bhopal 462066, India
}

(Received 19 August 2021; accepted 15 November 2021; published 7 December 2021)

\begin{abstract}
We take a bottom-up approach to find the interaction potential between the anti-de Sitter (AdS) black hole molecules under mean-field approximation. We start with the equation of state of dyonic AdS black holes in a fixed charge ensemble and use the method of classical cluster expansion to find the mean-field potential. We show that the Lennard-Jones (LJ) potential is a feasible choice to describe the equation of state. The LJ potential describes a two-body interaction. There exists a critical distance $r_{0}$ such that two interacting particles repel (attract) each other for $r<r_{0}\left(r>r_{0}\right)$. We compute the value of $r_{0}$ for dyonic AdS black holes and compare the result obtained from the Ruppeiner scalar curvature. Our analysis shows how the electric (and magnetic) charge affects the interaction between black hole molecules.
\end{abstract}

DOI: 10.1103/PhysRevD.104.126009

\section{INTRODUCTION AND SUMMARY}

Black holes are believed to be thermal objects [1]. Different macroscopic variables of black holes are identified with those of standard thermodynamics and the relations between them resemble the laws of thermodynamics [2]. For example, the area of the event horizon, the ADM mass and the surface gravity of a black hole are identified with entropy, total energy and temperature respectively [3,4] and their differential changes satisfy a relation which is similar to the first law of thermodynamics. In order to confirm that the black holes are indeed thermal objects, one needs to understand the microscopic origin of the thermal structure. This is still a challenging and open problem in black hole physics. String theory has successfully provided a partial answer to this question in the context of supersymmetric extremal asymptotically flat black holes [5]. A comprehensive understanding of black hole microscopy for a generic class of black holes, including the anti-de Sitter (AdS) ones, is missing. In this paper, we try to shed some light towards the understanding of AdS black hole microstructure.

A surprising similarity between electrically charged AdS black holes and van der Waals fluid was first observed in [6]. The phase structure of a charged black hole in the fixed charge ensemble is similar to that of a van der Waals fluid

\footnotetext{
*suvankar@iiserb.ac.in †urmeet17@iiserb.ac.in
}

Published by the American Physical Society under the terms of the Creative Commons Attribution 4.0 International license. Further distribution of this work must maintain attribution to the author(s) and the published article's title, journal citation, and DOI. Funded by SCOAP ${ }^{3}$. (nonideal fluid) if one identifies the inverse temperature, electric charge and horizon radius of the black hole with pressure, temperature and volume of the liquid-gas system respectively $[6,7]$. Such resemblance was further studied by [8-10] with a different identification between the parameters. Following the earlier works [11-14], Kubiznak and Mann considered the negative cosmological constant to be thermodynamic pressure of the AdS black hole and volume covered by the event horizon to be thermodynamic volume conjugate to pressure [15-18]. This gives a one to one mapping between charges AdS black holes and van der Waals fluid. Although such identification does not explain anything about the microstructure, this was an important observation towards the microscopy of an AdS black hole.

Wei and Liu proposed that the thermal AdS black holes have microstructure called black hole molecules [19]. Their claim was based on the similarity between the black hole phase structure and that of a van der Waals fluid. Identifying the horizon radius with the specific volume, Wei and Liu introduced the idea of number density of black hole molecules (the inverse of specific volume) to measure the microscopic degrees of freedom. They observed that the number density suffers a sudden change accompanied by a latent heat when the black hole undergoes a phase transition. Calculating the Ruppeiner scalar curvature, they also showed that there is a weak attractive interaction between two black hole molecules. However, the origin of such microstructure is not clear.

Ruppeiner developed a geometric interpretation of a thermal system by constructing a thermodynamic line element (or metric) in a space (thermodynamic manifold) spanned by the thermodynamic variables [20-22]. Following the standard techniques of Riemannian geometry, Ruppeiner 
constructed a scalar curvature of the thermodynamic manifold. It turns out that the sign of the scalar curvature signifies the nature of interactions between the molecules of the system. In particular, the positive (negative) curvature implies repulsion (attraction) between the constituent molecules. ${ }^{1}$ The idea of Ruppeiner's construction was first implemented to a BTZ black hole in [23]. Application of this idea on a variety of black holes in AdS space can be obtained in [24-30]. Wei, Liu and Mann constructed the Ruppeiner scalar for charged AdS black holes in a temperature and volume plane and showed that the characteristic curves in the $(T, V)$ plane are similar to those of van der Waals fluid $^{2}$ except for some interesting corners. There are regions in the $(T, V)$ plane where the sign of the Ruppeiner scalar is negative (positive) denoting attractive (repulsive) interaction between black hole molecules. In [35] Miao and $\mathrm{Xu}$ conjectured that the empirical Lennard-Jones potential is a feasible candidate to provide a qualitative explanation of the interaction between black hole micromolecules. They also showed that the interaction forces due to the Lennard-Jones potential match that of the Ruppeiner scalar curvature.

In this paper we take a bottom-up approach to find the interaction potential between the black hole molecules. The equation of states of AdS black holes is similar to the van der Waals fluid, which describes the state of interacting particles under mean-field approximation. At the same time, the critical exponents for the AdS black hole evaluated on the equation of state also take the "meanfield" values. A natural question arises at this point whether there is an effective mean-field interaction between the black hole molecules of AdS black holes. To answer this question we start with the equation of state of an AdS black hole in fixed charge ensemble and use the method of classical cluster expansion [36,37] to find the potential. It turns out that the Lennard-Jones (LJ) potential is a feasible candidate to describe such interaction. Our analysis supports the conjecture proposed in [35]. The LJ potential describes a two-body interaction. It has two parameters: strength of the potential $\epsilon$ and the critical distance $r_{0}$ such that two interacting particles repel each other for $r<r_{0}$ (i.e., when they come close to each other) and attract for $r>r_{0}$. We compute the value of $\epsilon$ and $r_{0}$ for dyonic AdS black holes and compare the result with that obtained from the Ruppeiner scalar curvature. In particular, we first find the interaction potential for a pure AdS black hole. We observe that for a pure AdS black hole the depth of the potential is proportional to the temperature and the critical volume $v_{0} \sim r_{0}^{3}$ is inversely proportional to the temperature.

\footnotetext{
${ }^{1}$ See Appendix A for a detailed discussion.

${ }^{2}$ See also [31-34].
}

This result is in agreement with [30,31]. We then turn on the electric and magnetic charges (dyonic black hole). Calculation of cluster integrals in the presence of generic electric and magnetic charges is complicated. In order to understand the effect of charges on the interaction between the molecules, we consider a small charge approximation. Our analysis shows that the strength of the potential and the critical volume reduces in the presence of electric (and magnetic) charges.

The paper is organized as follows:

(i) In Sec. II, we review the cluster expansion technique and compute the relation between virial coefficient and irreducible form of the cluster integrals.

(ii) In Sec. III, we discuss the thermodynamics and equations of state of the Schwarzschild black holes and the dyonic black holes in asymptotic AdS space-time.

(iii) In Sec. IV, we construct the mean-field potential by equating the cluster integrals with the virial coefficients. It turns out that the Lennard-Jones potential is a feasible candidate to describe the interaction between the black hole molecules.

(iv) Finally we summarize and discuss the results in Sec. V.

(v) In Appendix A we present the construction of Ruppenier curvature scalar for dyonic AdS black hole and discuss the relation between the signature of Ruppenier scalar and the nature of interaction between black hole molecules.

(vi) In Appendix B we compute the first few cluster integrals required in our computation.

\section{CLUSTER EXPANSION FOR NONIDEAL GAS}

Cluster expansion is a useful technique to compute the virial coefficient for an interacting gas of particles. The method was introduced by Mayer and Ursell. We use this technique to find the mean-field potential for black hole molecules. In this section, we briefly review the method. For details follow [36-38].

Consider a system of $N$ interacting particles of mass $m$ in volume $V$ and at temperature $T$. The Hamiltonian of the system is given by

$$
\mathcal{H}=\sum_{i=1}^{N} \frac{\mathbf{p}_{i}^{2}}{2 m}+\mathcal{V}\left(\mathbf{q}_{1}, \mathbf{q}_{2}, \ldots, \mathbf{q}_{N}\right) .
$$

Here $\mathbf{p}_{i}$ is the momentum of the $i$ th particle and the total potential $\mathcal{V}$ is given by

$$
\mathcal{V}\left(\mathbf{q}_{1}, \mathbf{q}_{2}, \ldots, \mathbf{q}_{N}\right)=\sum_{i<j} v_{i j}\left(\mathbf{q}_{i}-\mathbf{q}_{j}\right),
$$

where $v_{i j}$ is the potential between $i$ th and $j$ th particles which depends on their positions $\mathbf{q}_{i}$ and $\mathbf{q}_{j}$. Furthermore, the grand canonical partition function is given by 


$$
\begin{aligned}
\mathcal{L}(z, V, T) & =\sum_{N=0}^{\infty} z^{N} Q_{N}(V, T) \\
\text { where } Q_{N}(V, T) & =\frac{1}{N ! \hbar^{3 N}} \int d^{3 N} \mathbf{p} d^{3 N} \mathbf{q} \exp \left\{-\beta \sum_{i=1}^{N} \frac{\mathbf{p}_{i}^{2}}{2 m}-\beta \sum_{i<j} v_{i j}\left(\mathbf{q}_{i}-\mathbf{q}_{j}\right)\right\}
\end{aligned}
$$

where $\beta=\frac{1}{k_{B} T}$ is the inverse temperature and $z$ is the fugacity. The integral over the momenta can be done and the final result is given by

$$
\begin{aligned}
\mathcal{L}(z, V, T) & =\sum_{N=0}^{\infty}\left(\frac{z}{\lambda^{3}}\right)^{N} \frac{Z_{N}(V, T)}{N !} \\
\text { where } Z_{N}(V, T) & =\int d^{3 N} q \exp \left(-\beta \sum_{i<j} v_{i j}\right) .
\end{aligned}
$$

Here $\lambda=\sqrt{2 \pi \hbar^{2} / m k T}$ is the thermal wavelength. The integral in (2.4) is known as configuration integral (CI).

$\mathrm{CI}$ can be solved by using the technique of classical cluster expansion. Defining a Mayer's function $f_{i j}=$ $e^{-\beta v_{i j}}-1$ the CI can be written as

$$
Z_{N}(V, T)=\int d^{3 N} q \prod_{i<j}\left(1+f_{i j}\right)
$$

In order to solve the above integral one defines an $\ell$-linked cluster as a connected (by single lines) diagram of $\ell$ nodes. An $\ell$-cluster cannot be reduced into smaller clusters without cutting a single line. For example $f_{i j} f_{j k}$ denotes a single cluster of three nodes (three-cluster) carrying numbers $i, j$ and $k$ where the node $j$ is connected to both $i$ and $k$ but the later two are not connected. One can also define an $\ell$-linked cluster integral $b_{\ell}$ :

$$
b_{\ell}=\frac{1}{\ell ! \lambda^{3 \ell-3} V} \int d \mathbf{q}_{1} \cdots d \mathbf{q}_{\ell} \sum_{\ell \geq i>j \geq 1}^{\tilde{N}} \prod f_{i j}
$$

where $\tilde{\sum}$ denotes the sum over all the products of $f_{i j}$ consistent with an $\ell$-cluster as defined above. At thermal equilibrium the thermodynamic quantities pressure and density of the system can be written in terms of cluster integrals as

$$
\frac{P}{k_{B} T}=\frac{1}{\lambda^{3}} \sum_{\ell=1}^{\infty} \hbar_{\ell} z^{\ell}, \quad \frac{N}{V}=\frac{1}{v}=\frac{1}{\lambda^{3}} \sum_{\ell=1}^{\infty} \ell \hbar_{\ell} z^{\ell}
$$

where

$$
\hbar_{\ell}(T)=\lim _{V \rightarrow \infty} b_{\ell}(V, T)
$$

From Eq. (2.7) one can find that the virial expansion of equation of state is given by

$$
\frac{P v}{k_{B} T}=\sum_{\ell=1}^{\infty} a_{\ell}(T)\left(\frac{\lambda^{3}}{v}\right)^{\ell-1}
$$

where the virial coefficients $a_{\ell}$ are determined in terms of cluster integrals from the following identity:

$$
\left(b_{1} z+2 b_{2} z^{2}+3 b_{3} z^{3}+\cdots\right)\left[a_{1}+a_{2}\left(\sum_{n=1}^{\infty} n b_{n} z^{n}\right)+a_{3}\left(\sum_{n=1}^{\infty} n b_{n} z^{n}\right)^{2}+\cdots\right]=b_{1} z+b_{2} z^{2}+b_{3} z^{3}+\cdots
$$

The first few of them are given by

$$
a_{1}=b_{1}=1, \quad a_{2}=-\hbar_{2}, \quad a_{3}=4 \hbar_{2}^{2}-2 \hbar_{3}, \quad a_{4}=-20 b_{2}^{3}+18 b_{2} \hbar_{3}-3 b_{4}, \quad \ldots
$$

\section{A. Irreducible integral form of cluster integral}

The cluster integrals (2.6) are reducible. This means that the integrals can be written as a product of smaller integrals. Therefore one can further define irreducible cluster integrals $\beta_{k}$ :

$$
\beta_{k}=\frac{1}{k ! V} \int \cdots \int d \mathbf{q}_{1} \cdots d \mathbf{q}_{k+1} \sum_{k+1 \geq i>j \geq 1}^{-} \prod f_{i j},
$$

where $\bar{\sum}$ denotes the sum over all $k+1$ clusters which are more than singly connected. One cannot reduce such cluster into a smaller one by cutting a single line. Using the definitions of (2.6) and (2.11) the cluster integrals $b_{\ell}$ can be written in terms of irreducible cluster integrals $\beta_{k}$. The first few relations are given $b y^{3}$

\footnotetext{
${ }^{3}$ There exists a generic relation between $b_{\ell}$ and $\beta_{k}$. See [37].
} 


$$
\begin{aligned}
b_{1}=1, \quad b_{2} & =\frac{1}{2} \beta_{1}, \quad b_{3}=\frac{1}{2} \beta_{1}^{2}+\frac{1}{3} \beta_{2}, \\
b_{4} & =\frac{2}{3} \beta_{1}^{3}+\beta_{1} \beta_{2}+\frac{1}{4} \beta_{3}, \ldots
\end{aligned}
$$

Finally the equation of state for nonideal fluid can be written in terms of irreducible integrals:

$$
\frac{P v}{k_{B} T}=1-\sum_{k \geq 1} \frac{k}{k+1} \beta_{k}\left(\frac{1}{v}\right)^{k} .
$$

Comparing (2.9) and (2.13) one can find relations between virial coefficients and irreducible cluster integrals:

$a_{2}=-\frac{1}{2} \beta_{1}, \quad a_{3}=-\frac{2}{3} \beta_{2}, \quad a_{4}=-\frac{3}{4} \beta_{3} \cdots$.

\section{THERMODYNAMICS OF AdS DYONIC BLACK HOLES AND EQUATION OF STATE}

To derive the equation of state of a dyonic AdS black hole in $3+1$ dimensions in different ensembles we start with the Reissner-Nordström action,

$$
S_{\mathrm{EM}}=\frac{1}{16 \pi G} \int d^{4} x \sqrt{-g}\left(\mathcal{R}-\mathcal{F}^{2}-\frac{6}{L^{2}}\right),
$$

where $\mathcal{R}$ is the Ricci scalar, $\mathcal{F}$ is the $U(1)$ field strength and $L$ is the radius of AdS space that is related to the cosmological constant $\Lambda=-\frac{3}{L^{2}}$. The equation of motion obtained from this action admits a spherically symmetric solution,

$$
d s^{2}=-f(r) d t^{2}+\frac{d r^{2}}{f(r)}+r^{2}\left(d \theta^{2}+\sin ^{2} \theta d \varphi^{2}\right),
$$

where $f(r)=1+\frac{r^{2}}{L^{2}}-\frac{2 M}{r}+\frac{q_{e}^{2}+q_{m}^{2}}{r^{2}}$,

$$
A_{\mu} d x^{\mu}=\left(-\frac{q_{e}}{r}+\frac{q_{e}}{r_{+}}\right) d t+\left(q_{m} \cos \theta\right) d \varphi,
$$

where the integration constants $M, q_{e}$ and $q_{m}$ carry physical meaning of mass, electric and magnetic charges respectively. $A_{\mu}$ is the $U(1)$ gauge field and $r_{+}$is the position of the outer horizon: $f\left(r_{+}\right)=0$. The asymptotic value of the time component of the gauge field is considered to be electric potential of the system:

$$
\Phi_{e} \sim \frac{q_{e}}{r_{+}} .
$$

In order to study the thermodynamics and phase structure of the system one first chooses an ensemble. One can either choose fixed electric potential and magnetic charge ensemble $[39,40]$ or fixed electric and magnetic charge ensemble. The Hawking temperature in fixed charge ensemble is given by

$$
T=\frac{1}{\beta}=\frac{1}{4 \pi r_{+}}\left[1+\frac{3 r_{+}^{2}}{L^{2}}-\frac{q_{e}^{2}+q_{m}^{2}}{r_{+}^{2}}\right] .
$$

In our analysis we consider the cosmological constant $\Lambda=$ $-6 / L^{2}$ as thermodynamic pressure of the system which is different than the standard treatment. The idea of considering cosmological constant as thermodynamic pressure was first introduced $\mathrm{in}^{4}[8]$, i.e.,

$$
P=-\frac{\Lambda}{8 \pi}=\frac{3}{8 \pi} \frac{1}{L^{2}}
$$

since in the presence of a cosmological constant the first law of black hole thermodynamics becomes inconsistent with the Smarr relation unless the variation of $\Lambda$ is included in the first law. Once we consider variation of $\Lambda$ in the first law, the black hole mass $M$ is identified with enthalpy rather than internal energy of the system $[8,10,11]$.

Following [39,41] one can compute the free energy of AdS dyonic black holes in a fixed electric charge ensemble:

$$
W=\frac{I}{\beta}=\frac{1}{4 G_{4}}\left[r_{+}+\frac{3\left(q_{e}^{2}+q_{m}^{2}\right)}{r_{+}}-\frac{8 \pi P r_{+}^{3}}{3}\right] .
$$

The other thermodynamic variables are given by

$$
\begin{aligned}
\Phi_{e} & =\frac{\partial W}{\partial q_{e}}=\frac{q_{e}}{G_{4} r_{+}}, \quad \Phi_{m}=\frac{\partial W}{\partial q_{m}}=\frac{q_{m}}{G_{4} r_{+}}, \\
S & =-\frac{\partial W}{\partial T}=\beta^{2} \frac{\partial W}{\partial \beta}=\frac{1}{4 G_{4}} 4 \pi r_{+}^{2}=\frac{A_{H}}{4 G_{4}}, \\
V & =\frac{\partial W}{\partial P}=\frac{4 \pi}{3} r_{+}^{3} .
\end{aligned}
$$

Using Eq. (3.5) one can write the pressure as a function of $T, r_{+}, q_{e}$ and $q_{m}$ :

$$
\frac{P v}{T}=1-\frac{1}{2 \pi T} \cdot \frac{1}{v}+\frac{2\left(q_{e}^{2}+q_{m}^{2}\right)}{\pi T} \cdot \frac{1}{v^{3}},
$$

where $v=2 r_{+}$can be identified with the specific volume of the system. The above relation is the equation of state of a dyonic black hole in a fixed charge ensemble. One can also work on a fixed electric potential ensemble to start with [39]. The equation of state in that ensemble takes the form

$$
\frac{P v}{T}=1-\frac{1-\Phi_{e}^{2}}{2 \pi T} \cdot \frac{1}{v}+\frac{2 q_{m}^{2}}{\pi T} \cdot \frac{1}{v^{3}} .
$$

\footnotetext{
${ }^{4}$ It was suggested in [11-14] that $\Lambda$ can be treated as a thermodynamic variable of the system.
} 
These equations describe different phases of a dyonic black hole in different ensembles. Both equations have surprising similarity with the van der Wall equation or virial expansion in general. However, there is a potential difference between these equations and virial expansion. Unlike virial expansion both Eqs. (3.9) and (3.10) truncate after third order. Our goal is to study the interaction between black hole molecules which gives rise to these equations of state.

\section{MEAN-FIELD POTENTIAL FOR BLACK HOLE MOLECULES}

The van der Waals equation qualitatively explains the equation of the state of nonideal fluid. The equation of state and the critical exponents for van der Waals fluid can be derived under a mean-field approximation. In mean-field theory, we assume that each particle moves independently in an average potential field offered by the other particles. The famous Lennard-Jones potential is a good choice of mean-field potential to explain the behavior of nonideal fluid qualitatively. The LJ potential has two terms: the repulsive term (proportional to $1 / r^{12}$ ) describes the Pauli repulsion at short distances between the molecules and the attractive term (proportional to $1 / r^{6}$ ) describes attraction at long distances.

The similarity between the equations of state of AdS black holes and van der Waals fluid and also the equality of the critical exponents of the two systems motivate one to postulate a microstructure for AdS black holes $[8,9,19]$. Further, computation of the Ruppeiner thermodynamic scalar for charged AdS black holes and van der Waals fluid also shows a surprising similarity between characteristic curves of both systems in the $(T, V)$ plane [31,42]. The characteristic curves contain information about the attractive and repulsive nature of the interaction between the molecules. Thus similarity of characteristic curves indicates that there could be a similar microstructure for AdS black holes. It has also been conjectured that the LJ potential qualitatively explains the phase structure of the AdS black hole [35]. In order to understand the underlying microstructure of AdS black holes in more detail, we try to derive the mean-field potential between the black hole molecules such that the corresponding equation of state is given by (3.9) or (3.10). Using the relations between virial coefficients and the irreducible cluster integrals (2.14) we find that the LJ potential is a feasible choice to explain the interaction. The LJ potential has a critical length denoted by $r_{0}$. If the distance between the molecules is greater (less) than that, then the interaction between them is attractive (repulsive). We find that the strength of the LJ potential can be adjusted such that the value of the critical length $r_{0}$ (for a given temperature and charges) matches with the result derived from the zeros of Ruppeiner curvature.

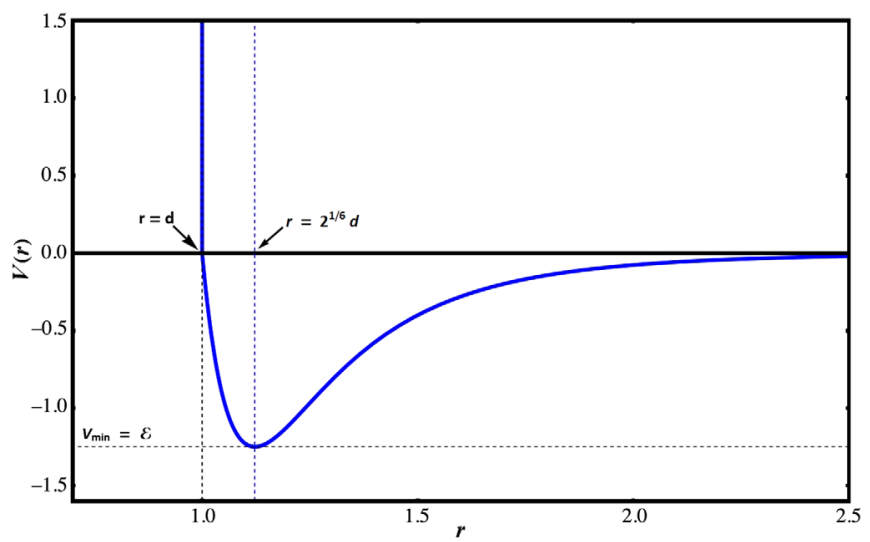

FIG. 1. Modified Lennard-Jones potential.

We find the following form of the potential suitably describes the interaction between AdS black hole molecules $^{5}$ :

$$
\mathcal{V}(r)= \begin{cases}\infty & \text { for } r<d \\ 4 \varepsilon\left[\left(\frac{d}{r}\right)^{12}-\left(\frac{d}{r}\right)^{6}\right] & \text { for } r \geq d .\end{cases}
$$

Here $r$ is the distance between two interacting molecules, $\varepsilon$ is the depth of the potential and $d$ is the distance at which the particle-particle interaction potential is zero. $d$ is proportional to the size of the molecules. In order to avoid the singular behavior at $r=0$ we consider hard sphere approximation, i.e., the potential is infinity for $r<d$. This implies that there exists a restricted volume $\omega=\frac{4 \pi}{3} d^{3}$ where no molecules can enter as the repulsion is infinity. For $r>d$ the potential has a minimum at

$$
r_{0}=2^{1 / 6} d
$$

From $r=d$ to $r=r_{0}$ the potential is repulsive and from $r=r_{0}$ to $\infty$ it is attractive. The minimum value of the potential is $\varepsilon$. See Fig. 1 .

The Mayer function under mean-field approximation takes the following form:

$f_{i j}(r)= \begin{cases}-1, & \text { for } r<d \\ \exp [-\beta \mathcal{V}(r)]-1 & \text { for } r \geq d, \quad \text { for all } i, j .\end{cases}$

\footnotetext{
${ }^{5}$ We modified the LJ potential for small $r$. The Lennard-Jones potential is a very rapidly increasing function of $r$ when $r$ is close to zero (it goes like $\sim 1 / r^{12}$ ). This implies a very strong repulsion between the molecules and hence two molecules coming in the vicinity of $r<d$ is extremely less probable. Therefore the modified potential is approximately equal to the Lennard-Jones potential. One can also work with the actual LJ potential. In that case the dependence of parameter $\epsilon$ on temperature (4.9) would remain the same up to a very small change in the numerical factors.
} 
We define a volume $v_{0}$ associated with the critical length $r_{0}$ :

$$
v_{0}=\frac{4 \pi}{3} r_{0}^{3} .
$$

Our goal is to compute this volume as a function of temperature and charge and compare the result obtained from the thermodynamic curvature [31].

Computation of (irreducible) cluster integrals for this potential is in general complicated. To get an analytic handle on the problem we make an approximation to simplify the Mayer function. We assume that $\beta \mathcal{V}(r)$ is small enough such that the Mayer function can be approximated as

$f_{i j}(r)= \begin{cases}-1, & \text { for } r<d \\ -4 \beta \varepsilon\left[\left(\frac{d}{r}\right)^{12}-\left(\frac{d}{r}\right)^{6}\right] & \text { for } r \geq d, \quad \text { for all } i, j .\end{cases}$

In what follows we see that in the high temperature limit this is a good approximation.

Using (2.6) and (2.11) we can write

$$
\begin{aligned}
\beta_{1} & =2 \hbar_{2}=\frac{1}{V} \iint d^{3} \mathbf{q}_{1} d^{3} \mathbf{q}_{2} f_{i j}\left(\mathbf{q}_{1}, \mathbf{q}_{2}\right) \\
& =-\frac{\beta}{V} \iint d^{3} \mathbf{q}_{1} d^{3} \mathbf{q}_{2} \mathcal{V}(r), \text { where } r=\left|\mathbf{q}_{1}-\mathbf{q}_{2}\right| .
\end{aligned}
$$

Going to the center of mass frame it is easy to do this integration and the final result is given by

$$
\beta_{1}=\left(-1+\frac{8 \varepsilon}{3 T}\right) \frac{v_{0}}{\sqrt{2}}
$$

We first consider a neutral AdS black hole and find the strength of the LJ potential $\varepsilon$ and the critical length $r_{0}$. The second virial coefficient $a_{2}$ for the neutral AdS black hole is given by (3.9):

$$
a_{2}=-\frac{1}{2 \pi T} .
$$

All other higher virial coefficients are zero. Comparing the second virial coefficient with the irreducible cluster integral $\beta_{1}$ one can choose

$$
\varepsilon=\frac{3}{8}(1+2 \sqrt{2}) T \sim 1.44 T
$$

such that $v_{0}$ is given by

$$
v_{0}=\frac{1}{2 \pi T} .
$$

In the high temperature limit this calculation is quite reliable since higher order irreducible cluster integrals are suppressed by higher powers of $1 / T$ :

$$
\beta_{k} \sim \frac{1}{T^{k}} \quad \text { for } k>1
$$

and hence the higher virial coefficients.

Thus we see that the LJ potential is a feasible candidate to describe a mean-field interaction between the black hole molecules in the high temperature limit. From the potential, we see that the interaction between two molecules is attractive if the specific volume $v$ is greater than the critical volume $v_{0}=1 / 2 \pi T$. The result is in agreement with the result obtained from the computation of the Ruppeiner thermodynamic scalar (see Appendix A). From the derivation, one can see that $v_{0} \sim 1 / T$ and $\epsilon \sim T$ up to some numerical values. The numerical value for $\epsilon$ can be adjusted such that (4.10) holds. Therefore from our calculation we see that the LJ potential (4.1) (up to some numerical factors) suitably describes the interaction between black hole molecules for AdS black holes. In the high temperature limit, the argument of the exponential in (4.3) goes as

$$
\beta \mathcal{V}(r) \sim \frac{1}{T^{2}}
$$

and hence our approximation $f_{i j}(r) \sim-\beta \mathcal{V}(r)$ is good enough.

\section{A. Dyonic black hole}

We next consider dyonic AdS black hole in a constant charge ensemble. ${ }^{6}$ The equation of state is given by (3.9). The virial coefficients are given by

$a_{1}=1, \quad a_{2}=-\frac{1}{2 \pi T}, \quad a_{3}=0, \quad a_{4}=\frac{2\left(q_{e}^{2}+q_{m}^{2}\right)}{\pi T}$

and all higher virial coefficients are zero. Calculation of irreducible cluster integrals in the presence of arbitrary electric and magnetic charges is tedious. We make a further (apart from the high temperature approximation) assumption here. We take the charges $q_{e}$ and $q_{m}$ to be small and keep terms up to order $q_{e}^{2}$ and $q_{m}^{2}$. We find the perturbative correction to the mean-field potential in the presence of charges. This helps us to understand how the electric and magnetic charges change the interaction between the black hole molecules.

We correct the ansatz for the Mayer function up to order $q^{2}$ where $q^{2}=q_{e}^{2}+q_{m}^{2}$ :

\footnotetext{
${ }^{6}$ The analysis for a fixed potential ensemble can be done in a similar way.
} 


$$
f_{i j}(r)= \begin{cases}-\left(1-q^{2} \delta\right), & \text { for } r<d \\ -4 \beta \varepsilon\left[\left(1+q^{2} \gamma\right)\left(\frac{d}{r}\right)^{12}-\left(1+q^{2} \sigma\right)\left(\frac{d}{r}\right)^{6}\right] & \text { for } r \geq d, \quad \text { for all } i, j,\end{cases}
$$

where $\delta, \gamma$ and $\sigma$ are constants and depend on temperature and charges. We compute the cluster integrals for the above potential. See Appendix B for details. Using the relation (2.14) we calculate the unknown coefficients $\delta, \gamma$ and $\sigma$. We see that there coefficients can be chosen as follows:

$$
\delta=\frac{2.65}{v_{0}^{2}}, \quad \sigma=\frac{19.58}{v_{0}^{2}} \quad \text { and } \quad \gamma=\frac{36.39}{v_{0}^{2}}
$$

such that the critical volume $v_{0}$ is given by

$$
v_{0}=\frac{1}{2 \pi T}-16 \pi T q^{2}
$$

which is in agreement of thermodynamic curvature calculation (A8). With these values one can calculate the minimum of the potential; it is given by

$$
\mathcal{V}_{\min }=-1.44 T\left(1-\frac{2.78 q^{2}}{v_{0}^{2}}\right)
$$

up to the order of $q^{2}$.

\section{DISCUSSION}

In this article, we probe the effect of electric and magnetic charges on the interaction between black hole molecules by calculating the effective mean-field interaction potential between them using the techniques of classical cluster expansion for a class of $3+1$ dimensional AdS dyonic black holes. The equation of state of the charged AdS black hole in the extended phase space is similar to that of a van der Waals fluid. To fully understand the microscopic interactions between the black hole molecules, we take a bottom-up approach to find the mean-field potential between them. It turns out that the Lennard-Jones potential is a feasible choice to explain the interaction between the molecules and it matches the result obtained by calculating the Ruppeiner thermal curvature.

We first find the LJ potential for a neutral AdS black hole. The computation of irreducible cluster coefficients, in general, is extremely complicated analytically. We consider the high temperature limit and compute these coefficients to find the mean-field potential. It turns out that if we choose the strength of the potential appropriately (the numerical factor), then the critical volume $v_{0}$ becomes $1 / 2 \pi T$. This implies that if the two black hole molecules come in a volume $v_{0}$ they start repelling each other; otherwise, there is an attraction between them. This result is in agreement with [30]. Our next goal was to understand the effect of the charge parameter $q$ at the microscopic level. Extending our approach for a generic charge parameter is again a formidable task to do. Therefore we make a further approximation. However, this allows us to understand the effect of charge on the microscopic interaction. It turns out that the charge $q$ reduces the size of the critical volume as well as the strength of the LJ potential. Correcting the Mayer (4.14) function for $r<d$ corresponds to deforming the hard sphere approximation in the presence of charges. The interaction potential for $r<d$ goes as $T \log \left(1 / q^{2}\right)$. In the limit $q \rightarrow 0$ the potential becomes $\infty$ for $r<d$.

The Ruppenier curvature scalar for dyonic black holes is given in Appendix A [Eq. (A.6b)]. From the expression we see that the curvature has another zero at $v_{0} \sim q^{2}$. Because of this extra zero the characteristic curves studied in [42] is different than the characteristic curves for a van der Waals fluid. However, in our analysis we do not see this extra solution for the critical volume $v_{0}$. This is because we studied the charged system as a perturbation over a pure AdS black hole. In the limit $q \rightarrow 0$ the second solution is $v_{0}=0$ and it did not show up in our calculation. An exact $q$ calculation might show the second solution for $v_{0}$.

From the microscopic point of view, different virial coefficients (2.9) account for multiparticle interactions. The $n$th virial coefficient can be calculated from the $n$-body interaction of the micromolecules. From the equation of state of a charged AdS black hole [(3.9) or (3.10)] we see that the microstructure for an AdS black hole is potentially different from the standard nonideal gas. The first term in the equation of state represents the ideal gas part. At a very high temperature $(T \rightarrow \infty)$ one can approximate the black hole molecules to be free gas. The second term in the equation implies that at finite temperature, the underlying molecules are not like ideal gas; rather, there is a repulsive two-body interaction between them. For a neutral AdS black hole, there are no multibody interactions between the molecules, whereas for a charged AdS black hole there are only two-body and four-body interactions at the microscopic level where the charge parameter $q$ accounts the strength of the four body interaction. The higher order virial coefficients appear under consideration of higher derivative terms in the action in higher dimensions [25,32,33,43]. However, we believe that the analysis can be extended to rotating black holes also. From the temperature of KerrAdS black holes one can write the equation of state and read the virial coefficients. It would be interesting to extend our computations for rotating black holes and compare the result with the same from Ruppeiner scalar computation $[25,44]$. From our analysis, we see that the four-body interaction is attractive in the limit of a small charge. 
Probing the microstructure via the equation of state does not allow us to count the number of microstates to match with the classical entropy. Consideration of black hole molecules is in some sense ad hoc. We presume that there exists some microstructure, but we exactly do not know the origin of such states. String theory, on the other hand, has been able to give a partial answer in order to construct the microstructure for macroscopic black holes. However, such black hole solutions are asymptotically flat and have a large amount of supersymmetry in order to compute the indices in the weak coupling limit [5]. An attempt to compute the microstructure for nonextremal black holes can be found in [45-47]. Computation of indices of supersymmetric field theories also allows one to understand the underlying microstates for the dual black holes in the context of the AdS/CFT correspondence. It would be interesting to explore if there is any connection between the ad hoc microstructure for supersymmetric AdS black holes and microstructures computed from supersymmetric indices.

\section{ACKNOWLEDGMENTS}

We would like to thank Robert Mann for useful discussion. The work of S. D. is supported by the MATRICS Grant No. MTR/2019/000390, the Department of Science and Technology, Government of India.

\section{APPENDIX A: RUPPEINER GEOMETRY}

Ruppeiner introduced a geometric interpretation of the thermal system introducing the thermodynamic line element in a space spanned by the macroscopic variables [20-22]. The line element defines the geometry of a manifold of thermodynamic variables. A thermodynamic system in equilibrium is expressed in terms of a set of macroscopic variables $\left\{x^{\mu}\right\}$. The infinitesimal distance between two neighboring thermal states is given by a thermodynamic line element. Ruppeiner showed that the several thermodynamic properties of the system were significantly encoded in the geometry of the thermodynamic manifold. The information of the phase structure of the system is captured by the Riemann scalar curvature of the thermodynamic manifold. Applying this idea to ideal Bose, Fermi, and classical ideal gas, one finds that the corresponding scalar curvature is positive, negative, and zero, respectively [48]. This observation suggests that the sign of scalar curvature can be used to understand the nature of interactions between the constituent particles of a thermodynamic system $[22,48,49]$. This is, therefore, in some sense, a bottom-up approach in contrast to the standard statistical mechanics.

In this Appendix, we provide a summary of Ruppenier's idea. Ruppenier geometry represents the covariant construction of the fluctuation theory of equilibrium thermodynamics. Fluctuation theory states that the neighboring fluctuation depends on the nearby thermodynamic parameters. The combination of the axioms of thermodynamic and fluctuation theorem leads to the line element $\Delta l^{2}$ for the distance between two neighboring fluctuations in thermal parameter space and is given by

$$
\Delta l^{2}=-\left.\frac{\partial^{2} S}{\partial x^{\alpha} \partial x^{\beta}}\right|_{x_{0}} \Delta x^{\alpha} \Delta x^{\beta}=-g_{\alpha \beta} \Delta x^{\alpha} \Delta x^{\beta},
$$

where $g_{\alpha \beta}=-\left.\frac{\partial^{2} S}{\partial x^{\alpha} \partial x^{\beta}}\right|_{x_{0}}$ and $x_{0}$ is the equilibrium point for thermal parameters and $g_{\alpha \beta}$ is the metric in thermal manifold in terms of thermodynamic entropy function $S\left(x, x_{0}\right)$. Then the line element (A1) in the thermal manifold is given by

$$
\Delta l^{2}=\frac{1}{T} \Delta T \Delta S-\frac{1}{T} \Delta P \Delta V+\sum_{i} \frac{1}{T} \Delta \mu_{i} \Delta N^{i} .
$$

\section{Ruppeiner geometry for black holes}

Here we apply the Ruppenier geometry for the AdS black hole where thermal parameter space is constructed with $(T, S, P, V)$, where $T$ is the Hawking temperature, $S$ is the entropy of the black hole, $P$ is the pressure of the AdS black hole associated with the cosmological constant and $V$ is the thermodynamic volume of the black hole. For $(T, V)$ fluctuation parameter space, the Helmholtz free energy $F$ is the governing potential for the thermal system, Helmholtz free energy can be calculated as $F=W-T S$, where $W$ is the Gibbs free energy given in Eq. (3.7).

The line element in Eq. (A.2) for $(T, V)$ fluctuation can be written as

$$
\Delta l^{2}=-\frac{1}{T}\left(\frac{\partial^{2} F}{\partial T^{2}}\right) \Delta T^{2}+\frac{1}{T}\left(\frac{\partial^{2} F}{\partial V^{2}}\right) \Delta V^{2} .
$$

As the specific heat at constant volume is defined as $C_{V}=T\left(\frac{\partial S}{\partial T}\right)_{V}=-T\left(\frac{\partial^{2} F}{\partial T^{2}}\right)_{V}$, the metric in terms of specific heat is

$$
\Delta l^{2}=\frac{C_{V}}{T^{2}} \Delta T^{2}-\frac{\left(\partial_{V} P\right)_{T}}{T} \Delta V^{2} .
$$

The specific heat at constant volume $C_{V}$ of the $\mathrm{AdS}$ black hole vanishes. As $C_{V}=T\left(\frac{\partial S}{\partial T}\right)_{V}$ and for the $(3+1)$-dimensional black hole case $S \propto A_{b h} \propto r_{h}{ }^{2}$ and $V \propto r_{h}{ }^{3}$, which implies $\left(\frac{\partial S}{\partial r_{h}}\right)_{V}=0$. The vanishing heat capacity $C_{V}$ implies that the temperature component of the line element in thermal geometry for a black hole $g_{T T}=0$ or $g^{T T}=\infty$. So we take vanishing heat capacity as the limit $k_{B} \rightarrow 0^{+}$, treating $C_{V}$ as a constant such that $C_{V} \rightarrow 0^{+}$. The scalar curvature diverges as specific heat tends to zero, so we modify the scalar curvature such that

$$
\mathcal{R}_{N}=\mathcal{R} C_{V} .
$$

The modified Ricci scalar for Eq. (A.4) geometry in terms of Hawking temperature and thermodynamic volume $V$ for Schwarzschild-AdS black hole and dyonic black hole is given by 


$$
\begin{gathered}
\mathcal{R}_{\text {SAdS }}^{(T V)}=\frac{1-2 \sqrt[3]{6} \pi^{2 / 3} T V^{1 / 3}}{2\left(\sqrt[3]{6} \pi^{2 / 3} T V^{1 / 3}-1\right)^{2}} \\
\mathcal{R}_{\text {Dyonic }}^{(T V)}=\frac{\left(8\left(q_{e}^{2}+q_{m}^{2}\right)-\left(\frac{6}{\pi}\right)^{2 / 3} V^{2 / 3}\right)\left(8\left(q_{e}^{2}+q_{m}^{2}\right)+12 T V-\left(\frac{6}{\pi}\right)^{2 / 3} V^{2 / 3}\right)}{2\left(8\left(q_{e}^{2}+q_{m}^{2}\right)+6 T V-\left(\frac{6}{\pi}\right)^{2 / 3} V^{2 / 3}\right)^{2}} .
\end{gathered}
$$

Solve the Ruppenier curvature for their vanishing point by inserting the thermodynamic volume $V$ in terms of specific volume $v$. The relation between thermodynamic volume and specific volume is $V=\pi v^{3} / 6$. First solve $\mathcal{R}_{\text {SAdS }}^{(T V)}=0$, which gives

$$
v_{0}=\frac{1}{2 \pi T}
$$

and then solve $\mathcal{R}_{\text {Dyonic }}^{(T V)}=0$ for small charge; we get

$$
v_{0}=\frac{1}{2 \pi T}-16 \pi T q^{2},
$$

where $q^{2}=q_{e}^{2}+q_{m}^{2}$.

\section{APPENDIX B: COMPUTATION OF CLUSTER INTEGRALS}

A general definition of irreducible integral is given in Eq. (2.11). Here we show the result for the second and third irreducible integrals, which we calculated for the potential ansatz given in Eq. (4.1). Explicitly, the $\beta_{2}$ integral is written as

$$
\beta_{2}=\frac{1}{2 ! V} \iiint f_{32} f_{31} f_{21} d \mathbf{q}_{1} d \mathbf{q}_{2} d \mathbf{q}_{3},
$$

where $f_{i j}=f\left(\left|\mathbf{q}_{i}-\mathbf{q}_{j}\right|\right)=e^{-\beta v_{i j}(r)}-1$ is the Mayer's function defined in (4.3). Under mean-field approximation, assuming $\beta \varepsilon \ll 1$ for Eq. (4.1), we get

$$
e^{-\beta v_{i j}(r)}-1 \approx-\beta v_{i j}(r) .
$$

To simplify the integral, we first change the integration variables such that $\mathbf{x}=\mathbf{q}_{1}-\mathbf{q}_{2}, \quad \mathbf{y}=\mathbf{q}_{1}-\mathbf{q}_{3}$, $\mathbf{z}=\frac{\mathbf{q}_{1}+\mathbf{q}_{2}+\mathbf{q}_{3}}{3}$, which implies $\mathbf{q}_{2}-\mathbf{q}_{3}=\mathbf{x}-\mathbf{y}$. Then the integral measure becomes

$$
d \mathbf{q}_{1} d \mathbf{q}_{2} d \mathbf{q}_{3}=d \mathbf{x} d \mathbf{y} d \mathbf{z} .
$$

We can easily integrate out the independent factors obtained from the change of variables. This reduces the integral in (B1) to a double integral of the form

$$
\beta_{2}=\frac{1}{2} \iint f(\|\vec{x}\|) f(\|\vec{y}\|) f(\|\vec{x}-\vec{y}\|) d \vec{x} d \vec{y} .
$$

Following Mayer's function from Eq. (4.3) along with the mean-field approximation, we can compute the integral as follows:

$$
\beta_{2} \approx \frac{\left(\omega^{2}\right)^{2}}{2}\left(-1-2.44 \frac{\varepsilon^{2}}{T^{2}}+3.25 \frac{\varepsilon^{3}}{T^{3}}\right) .
$$

Now, we can perform a similar simplification for the $\beta_{3}$ integral,

$$
\begin{aligned}
\beta_{3}= & \frac{1}{6 V} \iiint \int\left(3 f_{43} f_{32} f_{21} f_{41}+6 f_{43} f_{32} f_{21} f_{41} f_{31}\right. \\
& \left.+f_{43} f_{32} f_{21} f_{41} f_{31} f_{42}\right) d \mathbf{q}_{1} d \mathbf{q}_{2} d \mathbf{q}_{3} d \mathbf{q}_{4}
\end{aligned}
$$

using the change of variables:

$$
\begin{gathered}
\mathbf{x}=\mathbf{q}_{1}-\mathbf{q}_{2}, \quad \mathbf{y}=\mathbf{q}_{2}-\mathbf{q}_{3}, \quad \mathbf{z}=\mathbf{q}_{1}-\mathbf{q}_{4}, \\
\mathbf{w}=\frac{\mathbf{q}_{1}+\mathbf{q}_{2}+\mathbf{q}_{3}+\mathbf{q}_{4}}{4}
\end{gathered}
$$

which gives us $d \mathbf{q}_{1} d \mathbf{q}_{2} d \mathbf{q}_{3} d \mathbf{q}_{4}=d \mathbf{x} d \mathbf{y} d \mathbf{z} d \mathbf{w}$. Again, integrating out the independent factors in the integrand, we get

$$
\begin{aligned}
\beta_{3}= & \frac{1}{6} \iiint d \mathbf{x} d \mathbf{y} d \mathbf{z} f(|\mathbf{x}|) f(|\mathbf{y}|) f(|\mathbf{z}|) f(|\mathbf{x}+\mathbf{y}-\mathbf{z}|) \\
& \times\{3+6 f(|\mathbf{x}+\mathbf{y}|)+f(|\mathbf{x}+\mathbf{y}|) f(|\mathbf{x}-\mathbf{z}|)\} .
\end{aligned}
$$

Substituting the value of Mayer's function from Eq. (4.3) and assuming the approximation $x \gg d$, we can write $|x+y-z| \approx|x|,|x+y| \approx|x|,|x-z| \approx|x|$. Similarly, assuming $y \gg z \gg d$, we can approximate $|x+y-z| \approx|y|$, $|x+y| \approx|y|,|x-z| \approx|z|$. Using the potential ansatz from Eq. (4.1) and working in the mean-field approximation, the $\beta_{3}$ integral is evaluated as

$$
\begin{aligned}
\beta_{3} \approx & \frac{\omega^{3}}{3}\left(-1+1.83 \frac{\varepsilon^{2}}{T^{2}}-10.2 \frac{\varepsilon^{3}}{T^{3}}-5.28 \frac{\varepsilon^{4}}{T^{4}}\right. \\
& \left.+14.5 \frac{\varepsilon^{5}}{T^{5}}+1.82 \frac{\varepsilon^{6}}{T^{6}}\right) .
\end{aligned}
$$


[1] S. W. Hawking, Black holes and thermodynamics, Phys. Rev. D 13, 191 (1976).

[2] J. M. Bardeen, B. Carter, and S. W. Hawking, The four laws of black hole mechanics, Commun. Math. Phys. 31, 161 (1973).

[3] J. D. Bekenstein, Black holes and entropy, Phys. Rev. D 7, 2333 (1973).

[4] S. W. Hawking, Particle creation by black holes, Commun. Math. Phys. 43, 199 (1975); Erratum, Commun. Math. Phys. 46, 206 (1976).

[5] A. Strominger and C. Vafa, Microscopic origin of the Bekenstein-Hawking entropy, Phys. Lett. B 379, 99 (1996).

[6] A. Chamblin, R. Emparan, C. V. Johnson, and R. C. Myers, Charged AdS black holes and catastrophic holography, Phys. Rev. D 60, 064018 (1999).

[7] A. Chamblin, R. Emparan, C. V. Johnson, and R. C. Myers, Holography, thermodynamics and fluctuations of charged AdS black holes, Phys. Rev. D 60, 104026 (1999).

[8] D. Kubiznak and R. B. Mann, P-V criticality of charged AdS black holes, J. High Energy Phys. 07 (2012) 033.

[9] D. Kubiznak and R. B. Mann, Black hole chemistry, Can. J. Phys. 93, 999 (2015).

[10] D. Kubiznak, R. B. Mann, and M. Teo, Black hole chemistry: Thermodynamics with Lambda, Classical Quant. Grav. 34, 063001 (2017).

[11] D. Kastor, S. Ray, and J. Traschen, Enthalpy and the mechanics of AdS black holes, Classical Quant. Grav. 26, 195011 (2009).

[12] Y. Sekiwa, Thermodynamics of de Sitter black holes: Thermal cosmological constant, Phys. Rev. D 73, 084009 (2006).

[13] M. M. Caldarelli, G. Cognola, and D. Klemm, Thermodynamics of Kerr-Newman-AdS black holes and conformal field theories, Classical Quant. Grav. 17, 399 (2000).

[14] S. Wang, S.-Q. Wu, F. Xie, and L. Dan, The first laws of thermodynamics of the $(2+1)$-dimensional BTZ black holes and Kerr-de Sitter spacetimes, Chin. Phys. Lett. 23, 1096 (2006).

[15] E. Spallucci and A. Smailagic, Maxwell's equal area law for charged anti-de Sitter black holes, Phys. Lett. B 723, 436 (2013).

[16] N. Altamirano, D. Kubiž̌ák, R. B. Mann, and Z. Sherkatghanad, Kerr-AdS analogue of triple point and solid/liquid/ gas phase transition, Classical Quant. Grav. 31, 042001 (2014).

[17] R.-G. Cai, L.-M. Cao, L. Li, and R.-Q. Yang, P-V criticality in the extended phase space of Gauss-Bonnet black holes in AdS space, J. High Energy Phys. 09 (2013) 005.

[18] A. Dehyadegari, A. Sheykhi, and A. Montakhab, Critical behavior and microscopic structure of charged AdS black holes via an alternative phase space, Phys. Lett. B 768, 235 (2017).

[19] S.-W. Wei and Y.-X. Liu, Insight into the Microscopic Structure of an AdS Black Hole from a Thermodynamical Phase Transition, Phys. Rev. Lett. 115, 111302 (2015); Erratum, Phys. Rev. Lett. 116, 169903 (2016).

[20] G. Ruppeiner, Thermodynamics: A Riemannian geometric model, Phys. Rev. A 20, 1608 (1979).

[21] G. Ruppeiner, Thermodynamic Critical Fluctuation Theory?, Phys. Rev. Lett. 50, 287 (1983).
[22] G. Ruppeiner, Riemannian geometry in thermodynamic fluctuation theory, Rev. Mod. Phys. 67, 605 (1995); Erratum, Rev. Mod. Phys. 68, 313 (1996).

[23] R.-G. Cai and J.-H. Cho, Thermodynamic curvature of the BTZ black hole, Phys. Rev. D 60, 067502 (1999).

[24] J. E. Aman, I. Bengtsson, and N. Pidokrajt, Geometry of black hole thermodynamics, Gen. Relativ. Gravit. 35, 1733 (2003).

[25] J.E. Aman and N. Pidokrajt, Geometry of higherdimensional black hole thermodynamics, Phys. Rev. D 73, 024017 (2006).

[26] J.-y. Shen, R.-G. Cai, B. Wang, and R.-K. Su, Thermodynamic geometry and critical behavior of black holes, Int. J. Mod. Phys. A 22, 11 (2007).

[27] B. Mirza and M. Zamani-Nasab, Ruppeiner geometry of RN black holes: Flat or curved?, J. High Energy Phys. 06 (2007) 059.

[28] J. E. Åman and N. Pidokrajt, Ruppeiner geometry of black hole thermodynamics, EAS Publ. Ser. 30, 269 (2008).

[29] P. Chaturvedi, A. Das, and G. Sengupta, Thermodynamic geometry and phase transitions of dyonic charged AdS black holes, Eur. Phys. J. C 77, 110 (2017).

[30] Z.-M. Xu, B. Wu, and W.-L. Yang, Ruppeiner thermodynamic geometry for the Schwarzschild-AdS black hole, Phys. Rev. D 101, 024018 (2020).

[31] S.-W. Wei, Y.-X. Liu, and R. B. Mann, Ruppeiner geometry, phase transitions, and the microstructure of charged AdS black holes, Phys. Rev. D 100, 124033 (2019).

[32] S.-W. Wei and Y.-X. Liu, Extended thermodynamics and microstructures of four-dimensional charged GaussBonnet black hole in AdS space, Phys. Rev. D 101, 104018 (2020).

[33] A. Ghosh and C. Bhamidipati, Thermodynamic geometry for charged Gauss-Bonnet black holes in AdS spacetimes, Phys. Rev. D 101, 046005 (2020).

[34] A. Dehyadegari, A. Sheykhi, and S.-W. Wei, Microstructure of charged AdS black hole via $P-V$ criticality, Phys. Rev. D 102, 104013 (2020).

[35] Y.-G. Miao and Z.-M. Xu, On thermal molecular potential among micromolecules in charged AdS black holes, Phys. Rev. D 98, 044001 (2018).

[36] K. Huang, Statistical Mechanics, 2nd ed. (John Wiley \& Sons, New York, Chichester, Brisbane, Toronto, Singapore, 2008).

[37] J.E. Mayer and M. G. Mayer, Statistical Mechanics (John Wiley \& Sons, London, 1940).

[38] M. Kardar, Statistical Physics of Particles (Cambridge University Press, Cambridge, England, 2007).

[39] S. Dutta, A. Jain, and R. Soni, Dyonic black hole and holography, J. High Energy Phys. 12 (2013) 060.

[40] G.-J. Cheng, R.-R. Hsu, and W.-F. Lin, Dyonic black holes in string theory, J. Math. Phys. (N.Y.) 35, 4839 (1994).

[41] V. Balasubramanian and P. Kraus, A stress tensor for anti-de Sitter gravity, Commun. Math. Phys. 208, 413 (1999).

[42] S.-W. Wei, Y.-X. Liu, and R. B. Mann, Repulsive Interactions and Universal Properties of Charged Anti-de Sitter Black Hole Microstructures, Phys. Rev. Lett. 123, 071103 (2019).

[43] M. Cvetic, S. Nojiri, and S. D. Odintsov, Black hole thermodynamics and negative entropy in de Sitter and 
anti-de Sitter Einstein-Gauss-Bonnet gravity, Nucl. Phys. B628, 295 (2002).

[44] S.-W. Wei and Y.-X. Liu, A general thermodynamic geometry approach for rotating Kerr anti-de Sitter black holes, Phys. Rev. D 104, 084087 (2021).

[45] K. Sfetsos and K. Skenderis, Microscopic derivation of the Bekenstein-Hawking entropy formula for nonextremal black holes, Nucl. Phys. B517, 179 (1998).

[46] G. T. Horowitz, J. M. Maldacena, and A. Strominger, Nonextremal black hole microstates and $U$ duality, Phys. Lett. B 383, 151 (1996).
[47] J. C. Breckenridge, D. A. Lowe, R. C. Myers, A. W. Peet, A. Strominger, and C. Vafa, Macroscopic and microscopic entropy of near extremal spinning black holes, Phys. Lett. B 381, 423 (1996).

[48] H. Janyszek and R. Mrugaa, Riemannian geometry and stability of ideal quantum gases, J. Phys. A 23, 467 (1990).

[49] H. Janyszek, Riemannian geometry and stability of thermodynamical equilibrium systems, J. Phys. A 23, 477 (1990). 